\title{
A CENTRIFUGAL GRADOCOL FILTER
}

\author{
BY \\ S. D. ELEK AND G. R. F. HILSON \\ From the Department of Bacteriology, St. George's Hospital Medical \\ School, London
}

(RECEIVED FOR PUBLICATION SEPTEMBER 15, 1950)

Details of a centrifugal Seitz filter for sterilizing small quantities of fluid were reported some months ago in this journal (Hilson and Elek, 1950). It was then considered that a similar filter, fitted with a gradocol membrane (Elford, 1931) for virus or bacteriophage work where an asbestos pad is not suitable, might be useful. Moreover, a suggestion has been made (Latner and Slack, 1950) that such a device could also be used for the ultrafiltration of chemical mixtures. For these purposes a different design is necessary. The necessary requirements of such an apparatus are that it should be convenient for the handling of small quantities, since often the amount of available infectious material is limited. In addition it is essential that the apparatus should be safe in use, should not cause splashing, and should not leak. Since a wide range of pressures is needed when using gradocol membranes of different porosities both positive-pressure and suction types of apparatus are commonly employed. With centrifugal force, however, the whole range needed can easily be achieved.

The filter described here (Fig. 1) is suitable for use in standard laboratory centrifuges, in certain instances an adaptor collar being required. The capacity of the filter is about $4.5 \mathrm{ml}$. The upper part or cup has a flange at its upper end to fit the centrifuge collar, a pair of facets for a spanner, and at its lower end a thread on to which the lower part of the filter is screwed. The cup is closed with a screw-cap through which fluids may be introduced with a fine pipette ; its lower end forms a socket to receive a rubber washer, the gradocol disk, and its supporting perforated plate. Below the plate there is another rubber washer, and finally a pressure ring enters the socket and bears, through the washer, on the lower surface of the plate. This ring is prevented from rotation, relative to the socket and its contents, by means of a lateral lug which engages a slit in the side of the socket. The pressure ring has a funnel extension to direct the filtrate down the central axis, and it has a smooth bearing surface on its under side. The lower portion of the apparatus consists of a holder, having at its upper end a deep internal thread, a similar pair of spanner facets, and a stage with a smooth upper surface, which forms a rotating bearing with the pressure ring. Below this stage the holder contains a glass tube to receive the filtrate, and is closed at its lower end by a screw-cap. All those parts of the filter which will come in contact with fluid are made of stainless steel. 
For use, the holder is loaded with a glass tube and, together with the other metal parts, is sterilized by dry heat. The rubber washers are autoclaved, and filter disks of the right diameter are steam sterilized. The parts are then assembled, preferably under ultra-violet light. Alternatively, there seems to be little objection to assembling the filter loosely, with the membrane in position, and sterilizing by intermittent steaming. The filter is screwed up tight with spanners. As this is done, the stage bears on the pressure ring and drives it up the socket, so compressing the edge of the filter disk between the system of washers and perforated plate, and rendering the joints water-tight. The filters are centrifuged in pairs, and the required filtration pressure is obtained by adjusting the centrifuge speed according to a nomogram which correlates speed, distance of the disk from the axis, and the depth of fluid in the cup with the pressure developed (Fig. 2).

\section{Summary}

A centrifugal filter for use with gradocol membranes is described.

The filter is available in the size described and in a larger capacity model from Messrs. M.S.E., Ltd., 14, Spenser Street, London, S.W.1.
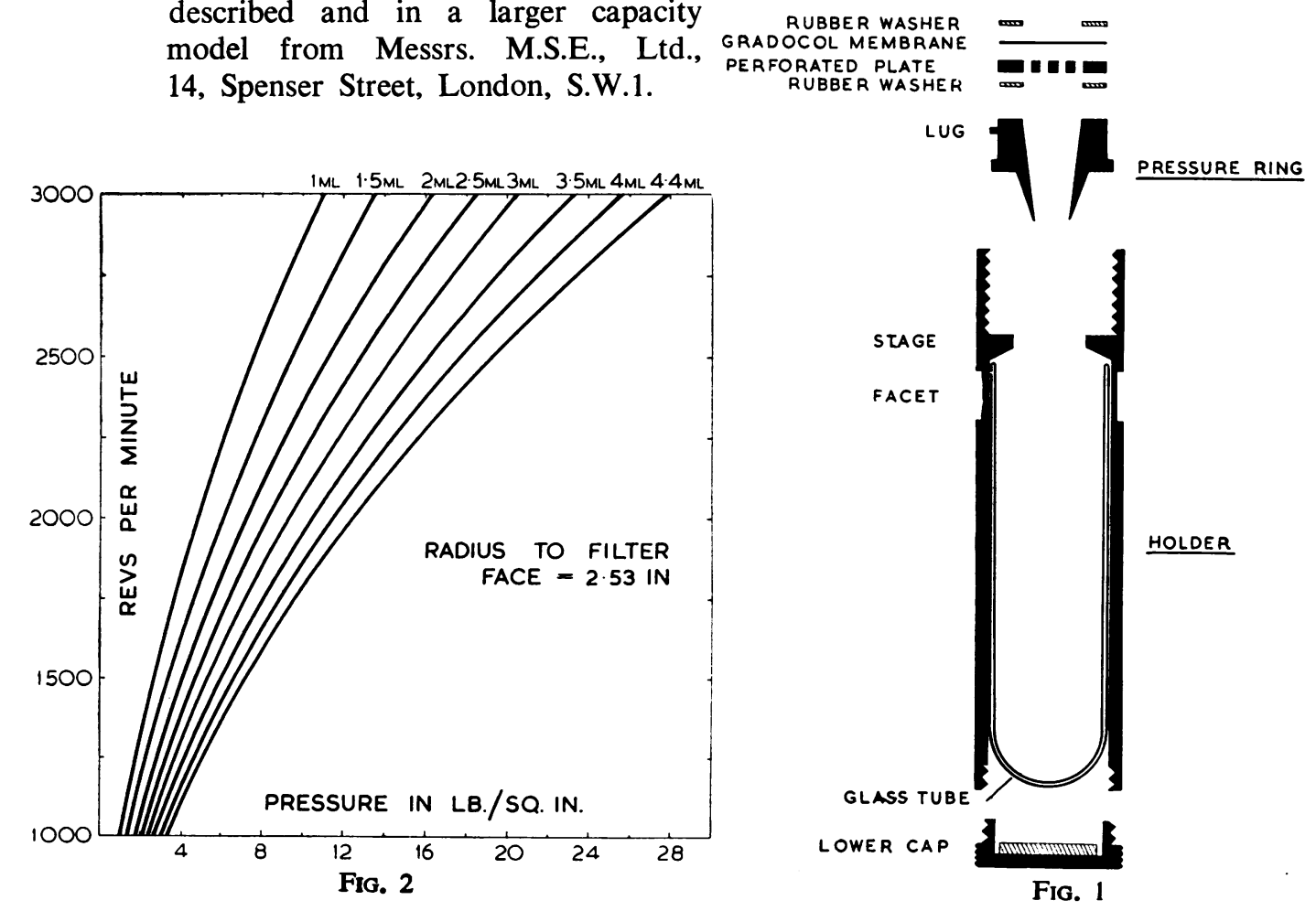
We wish to express our sincere thanks to Dr. W. J. Elford, F.R.S., of the Medical Research Council, for his kindness in testing the prototypes for us and for his valuable advice.

\section{REFERENCES}

Elford, W. J. (1931). J. Path. Bact., 34, 505.

Hilson, G. R. F., and Elek, S. D. (1950). J. clin. Path., 3, 67.

Latner, A. L., and Slack, E. B. (1950). Nature, Lond., 165, 530. 